\title{
Modified-release Granule for Oral Suspension
}

National Cancer Institute

\section{Source}

National Cancer Institute. Modified-release Granule for Oral Suspension. NCI Thesaurus. Code C149666.

Solid preparation consisting of modified-release granules intended to be dispersed in the specified liquid to obtain an oral suspension. 